\title{
Estudio del efecto de la adición de distintos mineralizadores en la síntesis cerámica del pigmento rosa coral de hierro-circón
}

\author{
M. LLUSAR, J. A. BADENES, J. CALBO, M. A. TENA, G. MONRÓS \\ Área de Química Inorgánica, U. P. de Química Inorgánica y Orgánica, Universidad Jaume I, Castellón, España
}

\begin{abstract}
Se ha analizado el efecto de la adición de distintos agentes mineralizadores (haluros alcalinos y alcalino-térreos, $\mathrm{KNO}_{3}$, etc.) en la síntesis cerámica tradicional del pigmento rosa coral de hierro-circón $\left(\mathrm{Fe}-\mathrm{ZrSiO}_{4}\right)$, usando dos precursores diferentes para el hierro (sulfato y goetita). El empleo de $\mathrm{FeSO}_{4} \cdot 7 \mathrm{H}_{2} \mathrm{O}$ como fuente de hierro permite obtener mejores coloraciones rojizas que con goetita $(\mathrm{FeO}(\mathrm{OH}))$. Los resultados obtenidos permiten clasificar a los distintos mineralizadores ensayados en dos grandes grupos: i) mineralizadores primarios o "formadores de circón", los cuales permiten activar el desarrollo del circón con una buena eficiencia en el encapsulamiento de la hematita en los cristales de circón $\left(\mathrm{NaF}, \mathrm{NaCl}\right.$ y $\mathrm{BaF}_{2}$ con $\mathrm{FeSO}_{4}, \mathrm{y} \mathrm{Na}_{2} \mathrm{SiF}_{6}$ y $\mathrm{NaAl}_{3} \mathrm{~F}_{6}$ con goetita), y ii) mineralizadores "secundarios o coadyuvantes", con un efecto colateral sobre la síntesis del pigmento, regulando

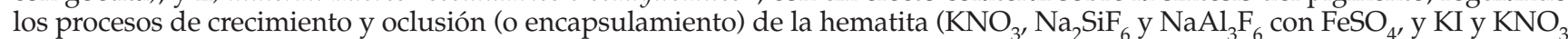
con goetita). Los mejores rendimientos colorimétricos han sido obtenidos con los sistemas mineralizadores binarios NaF$\mathrm{Na}_{2} \mathrm{SiF}_{6}, \mathrm{NaF}_{-} \mathrm{NaAl}_{3} \mathrm{~F}_{6}$ y NaF- $\mathrm{KNO}_{3}$.

Palabras clave: pigmento rosa coral, pigmento de hierro-circón, pigmentos de oclusión, mineralizadores, haluros.

Analysis of the effect of different mineralizers in the ceramic synthesis of the "pink coral" iron-zircon pigment

The addition of several mineralizing agents (alkaline or alkaline-earth halides, $\mathrm{KNO}_{3}$, etc.) in the traditional ceramic synthesis of the "coral pink" iron-zircon $\left(\mathrm{Fe}-\mathrm{ZrSiO}{ }_{4}\right.$ ) ceramic pigment has been analysed, using two different Fe precursors (sulphate and goethite). $\mathrm{FeSO}_{4} \cdot 7 \mathrm{H}_{2} \mathrm{O}$ employment as $\mathrm{Fe}$ precursor allows us to obtain a better reddish colour than with goethite $(\mathrm{FeO}(\mathrm{OH})$ ). Results let us to classify the studied mineralisers in two major groups: i) primary or zircon-forming mineralisers, which activate somehow zircon formation with a good occlusion efficiency of hematite into the zircon matrix $(\mathrm{NaF}, \mathrm{NaCl}$ and $\mathrm{BaF}_{2}$ when using $\mathrm{FeSO}_{4}$ and $\mathrm{Na}_{2} \mathrm{SiF}_{6}$ and $\mathrm{NaAl}_{3} \mathrm{~F}_{6}$ with goethite), and ii) secondary or modifying mineralisers, that have a collateral effect on the pigment synthesis regulating both hematite crystallisation and occlusion $\left(\mathrm{KNO}_{3}, \mathrm{Na}_{2} \mathrm{SiF}_{6}\right.$ and $\mathrm{NaAl}_{3} \mathrm{~F}_{6}$ with $\mathrm{FeSO}_{4}$ and $\mathrm{KI}$ and $\mathrm{KNO}_{3}$ with goethite). With regard to the color yield, those compositions mineralised with the binary systems $\mathrm{NaF}-\mathrm{Na}_{2} \mathrm{SiF}_{6}, \mathrm{NaF}^{-N_{a A l}} \mathrm{~F}_{6}$ and $\mathrm{NaF}-\mathrm{KNO}_{3}$ have been found to develop the best red color.
\end{abstract}

Keywords: pink coral, iron-zircon ceramic pigment, occlusion pigments, mineralizers, halides.

\section{INTRODUCCIÓN}

El pigmento rosa coral de hierro-circón, $(\mathrm{Zr}, \mathrm{Fe}) \mathrm{SiO}_{4}(\mathrm{DCMA}$ 14-44-5) (1), es uno de los tres pigmentos básicos del circón, junto con el azul de vanadio $(\mathrm{Zr}, \mathrm{V}) \mathrm{SiO}_{4}$ (DCMA 14-42-2) y el amarillo de praseodimio $(\mathrm{Zr}, \mathrm{Pr}) \mathrm{SiO}_{4}$ (DCMA 14-43-4). Estos pigmentos de circón son ampliamente utilizados en la industria de los pigmentos cerámicos (pueden suponer en torno al $50 \%$ de la producción global del sector) (2), debido a sus buenas prestaciones para aplicaciones de alta temperatura, dadas sus excelentes propiedades en cuanto a intensidad de color, brillo y estabilidad térmica (hasta $1380^{\circ} \mathrm{C}$ ).

La síntesis tradicional cerámica del rosa coral consiste en la mezcla u homogeneización del precursor de hierro correspondiente (generalmente $\mathrm{Fe}_{2} \mathrm{O}_{3}, \mathrm{FeO}(\mathrm{OH})$ o $\mathrm{FeSO}_{4} .7 \mathrm{H}_{2} \mathrm{O}$, etc.) con sílice y circona, y su posterior calcinación a temperaturas del orden de $900^{\circ} \mathrm{C}$ o incluso superiores. Asimismo, como sucede en la preparación de la mayoría de los pigmentos cerámicos, en la síntesis del rosa coral también se suelen emplear una amplia gama de compuestos, denominados mineralizadores (generalmente haluros), cuya función primordial consiste generalmente en mejorar la reactividad permitiendo la formación del pigmento a menores temperaturas y/o con menores tiempos de retención. En este sentido, el concepto de mineralizador se usa a veces indiscriminadamente o de una manera ambigua o confusa. De forma simplificada, los distintos agentes mineralizadores pueden ser clasificados en dos grandes grupos o familias, según la función que desempeñan en la síntesis del pigmento:

i) mineralizadores estructurales y/o cromomodificadores: son aquellos que contienen algún ion capaz de integrarse estructuralmente en la red huésped del pigmento produciendo un cambio en la coloración, ya sea al influir en la incorporación del agente cromóforo en dicha red o al modificar la intensidad del campo cristalino. Es el caso de los fluoruros que, según se cita en la bibliografía (3-5), pueden sustituir a los iones óxido en el pigmento azul de vanadio-circón, permitiendo una mayor incorporación de $\mathrm{V}^{4+}$ en la disolución sólida $\mathrm{V}^{4+}, \mathrm{F}^{-}-\mathrm{ZrSiO}_{4}$ y la obtención de coloraciones azul turquesa más intensas. También podrían incluirse en este grupo las sales de 
cerio añadidas a las espinelas de Fe-Cr o Fe-Ti, con el fin de desplazar hacia el rojo la coloración inicialmente marrón, o las sustituciones de $\mathrm{Al}$ por $\mathrm{Cr}$ en la perovsquita $\mathrm{NdAlO}_{3}$.

ii) mineralizadores promotores de la reactividad o adyuvantes: son todos aquellos compuestos capaces de mejorar la reactividad, efecto que puede conseguirse de muy diversa manera, bien sea por generación de fases fundentes en el transcurso de la reacción (empleo de fundentes clásicos como molibdatos, boratos, haluros alcalinos o alcalino-térreos, etc.) (6-9), por generación "in situ" de especies volátiles que movilicen algún componen-

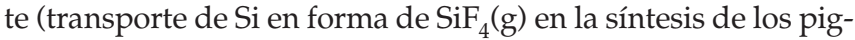
mentos de circón) (10-12), por "activación de la superficie" como consecuencia de los gases o vapores provenientes de las reacciones de descomposición de estos compuestos (caso del empleo de nitratos), o por control o estabilización del estado de oxidación del agente cromóforo (caso de la estabilización del $\mathrm{Fe}^{2+}$ con ioduros, sulfatos, policarboxilatos y otros quelatos, en la síntesis del pigmento rosa coral), por citar algunas posibilidades.

Por otra parte, a diferencia de los otros dos pigmentos de circón, en los que se forma una disolución sólida del cromóforo
(V o Pr) en la red de circón, en la bibliografía se considera ampliamente el pigmento rosa coral de hiero-circón como un pigmento encapsulado o de oclusión $(13,14)$, atribuyéndose el desarrollo del color a la encapsulación de la hematita en los cristales de circón. Sin embargo, esta afirmación aún es puesta en duda por algunos autores (15), quedando en todo caso por esclarecer el mecanismo exacto de formación del pigmento. A este respecto, Eppler et alter (15) atribuyen a una posible interacción de tipo superficial entre el $\mathrm{Fe}_{2} \mathrm{O}_{3}$ y el $\mathrm{ZrO}_{2}$, la presencia de una banda endotérmica en torno a $570^{\circ} \mathrm{C}$ en el análisis térmico diferencial de una mezcla equiponderal de circona, sílice y goetita en presencia de mineralizadores $\left(\mathrm{Na}_{2} \mathrm{SiF}_{6}\right.$ y $\left.\mathrm{BaSiF}_{6}\right)$; esta afirmación se sostiene de algún modo al constatarse un aumento en el rendimiento del color cuando se realizan precalcinas de la mezcla de estos dos reactivos a esta temperatura. Este resultado hace dudar a los autores de la naturaleza encapsulada del pigmento. En cambio, P. Tartaj et alter (16) no observan esta banda endotérmica en el análisis térmico de mezclas preparadas por pirólisis de aerosoles y en ausencia de mineralizadores, y sugieren un mecanismo de reacción en base a la formación de una disolución sólida de

TABLA I

EVOLUCIÓN CON LA TEMPERATURA DE CALCINACiÓN DE LAS FASES CRISTALINAS (DRX) PRESENTES EN LAS MUESTRAS $\left(\mathrm{SiO}_{2}\right)\left(\mathrm{ZrO}_{2}\right)_{0.97}\left(\mathrm{Fe}_{2} \mathrm{O}_{3}\right)_{0.15}$ (MINERALIZADOR) ${ }_{x}$, CON ADICIÓN SIMPLE DE MINERALIZAdOR (3\% PESO) Y UTILIZANDO FeSO $7_{4} 7 \mathrm{H}_{2} \mathrm{O}$ COMO FUENTE DE HIERRO.

\begin{tabular}{|c|c|c|c|c|c|c|}
\hline $\begin{array}{c}T\left({ }^{\circ} \mathrm{C}\right) \\
\text { Mineralizador }\end{array}$ & 850 & 900 & 950 & 1000 & 1050 & 1100 \\
\hline sin mineraliz. & $M(f) Q(f) H(d)$ & $\mathrm{M}(\mathrm{f}) \mathrm{Q}(\mathrm{f}) \mathrm{H}(\mathrm{d})$ & $M(f) Q(f) H(d)$ & $M(f) Q(f) H(d)$ & $M(f) Q(f) H(d)$ & $\mathrm{M}(\mathrm{f}) \mathrm{Q}(\mathrm{f}) \mathrm{H}(\mathrm{d})$ \\
\hline $\mathrm{NaF}$ & $\mathbf{Z}(\mathbf{f}) \mathrm{H}(\mathrm{d})$ & $\mathrm{Z}(\mathrm{f}) \mathrm{H}(\mathrm{d})$ & $Z(f) H(d)$ & $\mathbf{Z}(\mathrm{f}) \mathrm{H}(\mathrm{d})$ & $\mathbf{Z}(\mathbf{f}) \mathrm{H}(\mathrm{d})$ & $\mathbf{Z}(\mathbf{f}) \mathrm{H}(\mathrm{d})$ \\
\hline $\mathrm{NaCl}$ & $\mathrm{Z}(\mathrm{m}) \mathrm{M}(\mathrm{f}) \mathrm{H}(\mathrm{d})$ & $\mathrm{Z}(\mathrm{m}) \mathrm{M}(\mathrm{f}) \mathrm{H}(\mathrm{d})$ & $\mathrm{Z}(\mathrm{m}) \mathrm{M}(\mathrm{f}) \mathrm{H}(\mathrm{d})$ & $\mathrm{Z}(\mathrm{m}) \mathrm{M}(\mathrm{f}) \mathrm{H}(\mathrm{d})$ & $\mathrm{Z}(\mathrm{m}) \mathrm{M}(\mathrm{f}) \mathrm{H}(\mathrm{d})$ & $\mathrm{Z}(\mathrm{m}) \mathrm{M}(\mathrm{f}) \mathrm{H}(\mathrm{d})$ \\
\hline $\mathrm{KNO}_{3}$ & $\mathrm{Q}(\mathrm{m}) \mathrm{M}(\mathrm{f}) \mathrm{T}, \mathrm{H}(\mathrm{md})$ & $\mathrm{Q}(\mathrm{m}) \mathrm{M}(\mathrm{f}) \mathrm{T}, \mathrm{H}(\mathrm{md})$ & $\mathrm{Q}(\mathrm{d}) \mathrm{M}(\mathrm{f}) \mathrm{T}, \mathrm{H}(\mathrm{md})$ & $\mathrm{Q}(\mathrm{d}) \mathrm{M}(\mathrm{f}) \mathrm{T}, \mathrm{H}(\mathrm{md})$ & $\mathrm{Q}(\mathrm{md}) \mathrm{M}(\mathrm{f}) \mathrm{T}, \mathrm{H}(\mathrm{md})$ & $\mathrm{M}(\mathrm{f}) \mathrm{T}, \mathrm{H}(\mathrm{md})$ \\
\hline $\mathrm{BaF}_{2}$ & $\mathrm{Q}(\mathrm{d}) \mathrm{M}(\mathrm{f}) \mathrm{B}, \mathrm{H}(\mathrm{md})$ & $\mathrm{Z}(\mathrm{f}) \mathrm{M}(\mathrm{d}) \mathrm{B}, \mathrm{H}(\mathrm{md})$ & $\mathrm{Z}(\mathrm{f}) \mathrm{M}(\mathrm{d}) \mathrm{B}, \mathrm{H}(\mathrm{md})$ & $\mathrm{Z}(\mathrm{f}) \mathrm{M}(\mathrm{d}) \mathrm{B}, \mathrm{H}(\mathrm{md})$ & $\mathrm{Z}(\mathrm{f}) \mathrm{M}(\mathrm{d}) \mathrm{B}, \mathrm{H}(\mathrm{md})$ & $\mathrm{Z}(\mathrm{f}) \mathrm{M}(\mathrm{d}) \mathrm{B}, \mathrm{H}(\mathrm{md})$ \\
\hline $\mathrm{Na}_{3} \mathrm{AlF}_{6}$ & $\mathrm{Q}(\mathrm{m}) \mathrm{M}(\mathrm{mf}) \mathrm{H}(\mathrm{d})$ & $\mathrm{Q}(\mathrm{m}) \mathrm{M}(\mathrm{mf}) \mathrm{H}(\mathrm{d})$ & $\mathrm{Q}(\mathrm{m}) \mathrm{M}(\mathrm{mf}) \mathrm{H}(\mathrm{d})$ & $\mathrm{Q}(\mathrm{m}) \mathrm{M}(\mathrm{mf}) \mathrm{H}(\mathrm{d})$ & $\mathrm{Q}(\mathrm{m}) \mathrm{M}(\mathrm{mf}) \mathrm{H}(\mathrm{d})$ & $\mathrm{Q}(\mathrm{m}) \mathrm{M}(\mathrm{mf}) \mathrm{H}(\mathrm{d})$ \\
\hline $\mathrm{Na}_{2} \mathrm{SiF}_{6}$ & $\mathrm{Q}(\mathrm{m}) \mathrm{M}(\mathrm{mf}) \mathrm{H}(\mathrm{d})$ & $\mathrm{Q}(\mathrm{m}) \mathrm{M}(\mathrm{mf}) \mathrm{H}(\mathrm{d})$ & $\mathrm{Q}(\mathrm{m}) \mathrm{M}(\mathrm{mf}) \mathrm{H}(\mathrm{d})$ & $\mathrm{Q}(\mathrm{m}) \mathrm{M}(\mathrm{mf}) \mathrm{H}(\mathrm{d})$ & $\mathrm{Q}(\mathrm{m}) \mathrm{M}(\mathrm{mf}) \mathrm{H}(\mathrm{d})$ & $\mathrm{Q}(\mathrm{m}) \mathrm{M}(\mathrm{mf}) \mathrm{H}(\mathrm{d})$ \\
\hline
\end{tabular}

Fases cristalinas: $\mathrm{Q}$ (cuarzo, $\left.\mathrm{SiO}_{2}\right), \mathrm{M}\left(\mathrm{ZrO}_{2}\right.$ monoclínica), $\mathrm{T}\left(\mathrm{ZrO}_{2}\right.$ tetragonal), $\mathrm{Z}$ (circón, $\left.\mathrm{ZrSiO}_{4}\right), \mathrm{H}$ (hematita, $\left.\alpha-\mathrm{Fe}_{2} \mathrm{O}_{3}\right)$ y $\mathrm{B}$ (barita, BaSO ). Intensidad de los picos de $D R X: \mathrm{mf}$ (muy fuerte), $\mathrm{f}$ (fuerte), $\mathrm{m}$ (media), $\mathrm{d}$ (débil) and md (muy débil).

TABLA II

EVOLUCIÓN CON LA TEMPERATURA DE CALCINACIÓN DE LAS FASES CRISTALINAS (DRX) PRESENTES EN LAS MUESTRAS $\left(\mathrm{SiO}_{2}\right)\left(\mathrm{ZrO}_{2}\right)_{0.97}\left(\mathrm{Fe}_{2} \mathrm{O}_{3}\right)_{0.15}$

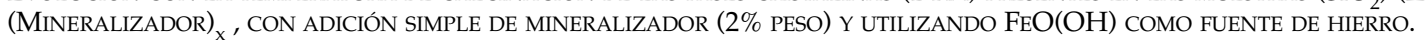

\begin{tabular}{|c|c|c|c|c|c|c|}
\hline $\begin{array}{c}\left.{ }^{\circ}{ }^{\circ} \mathrm{C}\right) \\
\text { Mineralizador }\end{array}$ & 850 & 900 & 950 & 1000 & 1050 & 1100 \\
\hline sin mineraliz. & $\mathrm{M}(\mathrm{f}) \mathrm{Q}(\mathrm{f}) \mathrm{H}(\mathrm{d})$ & $\mathrm{M}(\mathrm{f}) \mathrm{Q}(\mathrm{f}) \mathrm{H}(\mathrm{d})$ & $\mathrm{M}(\mathrm{f}) \mathrm{Q}(\mathrm{f}) \mathrm{H}(\mathrm{d})$ & $M(f) Q(f) H(d)$ & $\mathrm{M}(\mathrm{f}) \mathrm{Q}(\mathrm{f}) \mathrm{H}(\mathrm{d})$ & $\mathrm{M}(\mathrm{f}) \mathrm{Q}(\mathrm{f}) \mathrm{H}(\mathrm{d})$ \\
\hline $\mathrm{MgF}_{2}$ & $\mathrm{M}(\mathrm{f}) \mathrm{Q}(\mathrm{f}) \mathrm{H}(\mathrm{d})$ & $\mathrm{M}(\mathrm{f}) \mathrm{Q}(\mathrm{f}) \mathrm{H}(\mathrm{d})$ & $\mathrm{M}(\mathrm{f}) \mathrm{Q}(\mathrm{f}) \mathrm{H}(\mathrm{d})$ & $\mathrm{M}(\mathrm{f}) \mathrm{Q}(\mathrm{f}) \mathrm{H}(\mathrm{d})$ & $\mathrm{Z}(\mathrm{f}) \mathrm{M}, \mathrm{Q}$ (d) $\mathrm{H}(\mathrm{d})$ & $\mathbf{Z}(\mathrm{f}) \mathrm{M}, \mathrm{Q}(\mathrm{d}) \mathrm{H}(\mathrm{d})$ \\
\hline KI & $\mathrm{Q}(\mathrm{f}) \mathrm{M}(\mathrm{f}) \mathrm{H}(\mathrm{d})$ & $\mathrm{Q}(\mathrm{f}) \mathrm{M}(\mathrm{f}) \mathrm{H}(\mathrm{d})$ & $\mathrm{Q}(\mathrm{f}) \mathrm{M}(\mathrm{f}) \mathrm{H}(\mathrm{d})$ & $\mathrm{Q}(\mathrm{f}) \mathrm{M}(\mathrm{f}) \mathrm{H}(\mathrm{d})$ & $\mathrm{Q}(\mathrm{f}) \mathrm{M}(\mathrm{f}) \mathrm{H}(\mathrm{d})$ & $\mathrm{Q}(\mathrm{f}) \mathrm{M}(\mathrm{f}) \mathrm{H}(\mathrm{d})$ \\
\hline $\mathrm{KNO}_{3}$ & $\mathrm{Q}(\mathrm{f}) \mathrm{M}(\mathrm{f}) \mathrm{H}(\mathrm{d})$ & $\mathrm{Q}(\mathrm{f}) \mathrm{M}(\mathrm{f}) \mathrm{H}(\mathrm{d})$ & $\mathrm{Q}(\mathrm{f}) \mathrm{M}(\mathrm{f}) \mathrm{H}(\mathrm{d})$ & $\mathrm{Q}(\mathrm{f}) \mathrm{M}(\mathrm{f}) \mathrm{H}(\mathrm{d})$ & $\mathrm{Q}(\mathrm{f}) \mathrm{M}(\mathrm{f}) \mathrm{H}(\mathrm{d})$ & $\mathrm{Q}(\mathrm{f}) \mathrm{M}(\mathrm{f}) \mathrm{H}(\mathrm{d})$ \\
\hline $\mathrm{BaF}_{2}$ & $\mathrm{Q}(\mathrm{f}) \mathrm{M}(\mathrm{f}) \mathrm{H}(\mathrm{d})$ & $\mathrm{Q}(\mathrm{f}) \mathrm{M}(\mathrm{f}) \mathrm{H}(\mathrm{d})$ & $\mathrm{Q}(\mathrm{f}) \mathrm{M}(\mathrm{f}) \mathrm{H}(\mathrm{d})$ & $\mathrm{Q}(\mathrm{f}) \mathrm{M}(\mathrm{f}) \mathrm{H}(\mathrm{d})$ & $\mathrm{Q}(\mathrm{f}) \mathrm{M}(\mathrm{f}) \mathrm{H}(\mathrm{d})$ & $\mathrm{Q}(\mathrm{f}) \mathrm{M}(\mathrm{f}) \mathrm{H}(\mathrm{d})$ \\
\hline $\mathrm{Na}_{2} \mathrm{SiF}_{6}$ & $\mathrm{Q}(\mathrm{m}) \mathrm{M}(\mathrm{mf}) \mathrm{H}(\mathrm{d})$ & $\mathrm{Q}(\mathrm{m}) \mathrm{M}(\mathrm{mf}) \mathrm{H}(\mathrm{d})$ & $\mathrm{Q}(\mathrm{m}) \mathrm{M}(\mathrm{mf}) \mathrm{H}(\mathrm{d})$ & $\mathrm{Q}(\mathrm{m}) \mathrm{M}(\mathrm{mf}) \mathrm{H}(\mathrm{d})$ & $\mathrm{Q}(\mathrm{m}) \mathrm{M}(\mathrm{mf}) \mathrm{H}(\mathrm{d})$ & $\mathrm{Q}(\mathrm{m}) \mathrm{M}(\mathrm{mf}) \mathrm{H}(\mathrm{d})$ \\
\hline $\mathrm{Na}_{3} \mathrm{AlF}_{6}$ & $\mathrm{Q}(\mathrm{m}) \mathrm{M}(\mathrm{mf}) \mathrm{H}(\mathrm{d})$ & $\mathrm{Q}(\mathrm{m}) \mathrm{M}(\mathrm{mf}) \mathrm{H}(\mathrm{d})$ & $\mathrm{Q}(\mathrm{m}) \mathrm{M}(\mathrm{mf}) \mathrm{H}(\mathrm{d})$ & $\mathrm{Q}(\mathrm{m}) \mathrm{M}(\mathrm{mf}) \mathrm{H}(\mathrm{d})$ & $\mathrm{Q}(\mathrm{m}) \mathrm{M}(\mathrm{mf}) \mathrm{H}(\mathrm{d})$ & $\mathrm{Q}(\mathrm{m}) \mathrm{M}(\mathrm{mf}) \mathrm{H}(\mathrm{d})$ \\
\hline
\end{tabular}

Fases cristalinas: $\mathrm{Q}$ (cuarzo, $\mathrm{SiO}_{2}$ ), $\mathrm{M}\left(\mathrm{ZrO}_{2}\right.$ monoclínica), $\mathrm{Z}$ (circón, $\mathrm{ZrSiO}_{4}$ ) y H (hematita, $\alpha-\mathrm{Fe}_{2} \mathrm{O}_{3}$ ). Intensidad de los picos de DRX: mf (muy fuerte), $\mathrm{f}$ (fuerte), $\mathrm{m}$ (media) y d (débil). 
hierro en circona tetragonal (basándose en medidas realizadas de los parámetros reticulares), de modo que la formación de los cristales de circón viene acompañada de la segregación del $\mathrm{Fe}^{3+}$ del retículo de la circona y de la transformación de $\gamma-\mathrm{Fe}_{2} \mathrm{O}_{3}$ (también detectada por espectroscopia Mössbauer) en hematita $\left(\alpha-\mathrm{Fe}_{2} \mathrm{O}_{3}\right)$.

Asumiendo la naturaleza de pigmento de oclusión del rosa coral, en la Figura 1 se propone un modelo general para explicar el proceso de encapsulación de la hematita: las finas partículas de hematita estarían distribuidas al azar junto a las partículas más groseras de circona y sílice; con el tratamiento térmico, la sílice en contacto con la circona reaccionaría en un mecanismo binario de difusión para formar circón, que sinterizaría con otros granos encapsulando las partículas de hematita, que permanecieron en su lugar o bien pudieron crecer durante el proceso anterior por "coarsening" (engrosamiento) difusivo. En consecuencia, tanto la formación del circón como la oclusión de la hematita deben tener lugar con un adecuado sincronismo para que el encapsulamiento de ésta sea eficaz y pueda conseguirse un rendimiento de color aceptable. Si éste no es el caso, ya sea por un crecimiento demasiado rápido de la hematita o del circón, las partículas de hematita no quedarían protegidas o encapsuladas de manera eficiente por los cristales de circón neoformados (quedando como hematita libre), obteniéndose coloraciones marrones en lugar del color rojizo o rosa característico.

Según este modelo, los procesos difusivos involucrados en las interacciones $\mathrm{Fe}-\mathrm{Zr}$, Fe-Si y/o Si-Zr y en el crecimiento de las partículas de hematita, dependerán en gran medida del tipo de precursor de hierro utilizado, de la granulometría de las partículas, así como del sistema mineralizador empleado, teniendo estas variables una influencia muy relevante sobre la síntesis del pigmento. El sistema mineralizador más idóneo será aquel que facilite la reacción de formación del circón sin producir un crecimiento excesivo de la hematita. Con estas consideraciones, en el presente trabajo se ha realizado un aná-

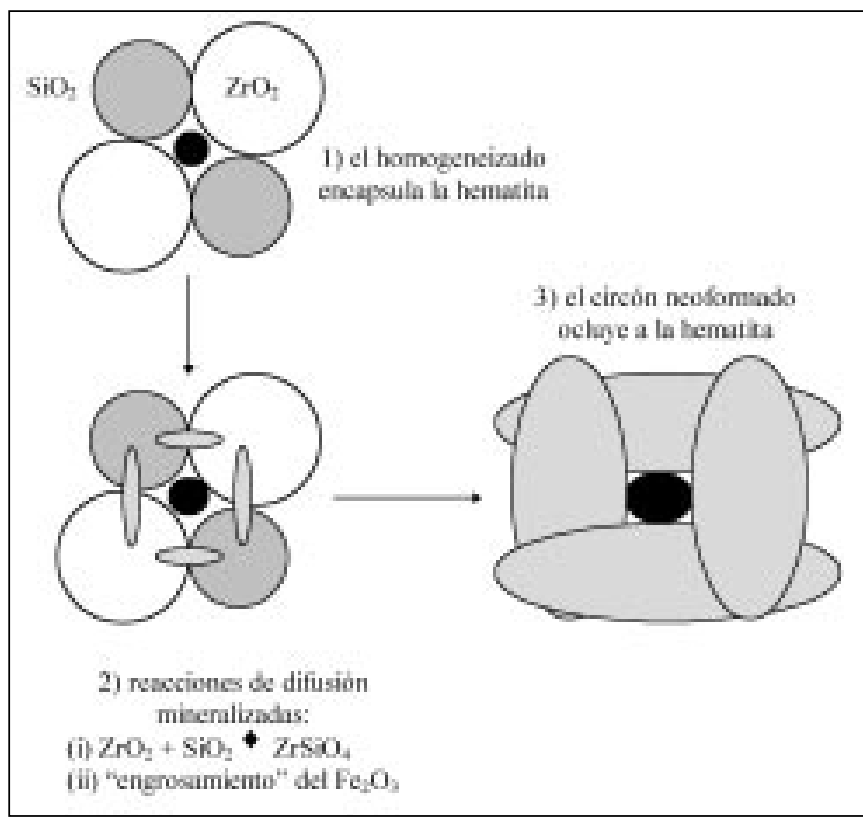

Figura 1. Modelo de "oclusión-crecimiento" para el encapsulamiento de la hematita en los cristales circón.

lisis sistemático del efecto de la adición de distintos mineralizadores sobre la síntesis del pigmento rosa coral, utilizando dos precursores diferentes para el hierro (sulfato y goetita).

\section{PROCEDIMIENTO EXPERIMENTAL.}

Utilizando el método tradicional cerámico se prepararon distintas composiciones de estequiometría general $\left(\mathrm{SiO}_{2}\right)$ $\left(\mathrm{ZrO}_{2}\right)_{0,97}\left(\mathrm{Fe}_{2} \mathrm{O}_{3}\right)_{0,15}(\mathrm{M})_{\mathrm{x}^{\prime}}$ donde $\mathrm{M}$ representa el sistema

TABLA III

EVOLUCIÓN CON LA CANTIDAD DE MINERALIZADOR $(2,5,8$ Y $12 \%$ EN PESO) DE LAS FASES CRISTALINAS (DRX) PRESENTES Y DEL COLOR EN LAS MUESTRAS

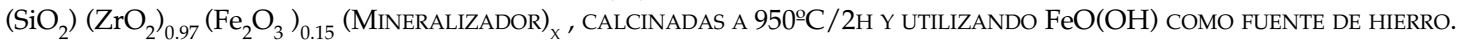

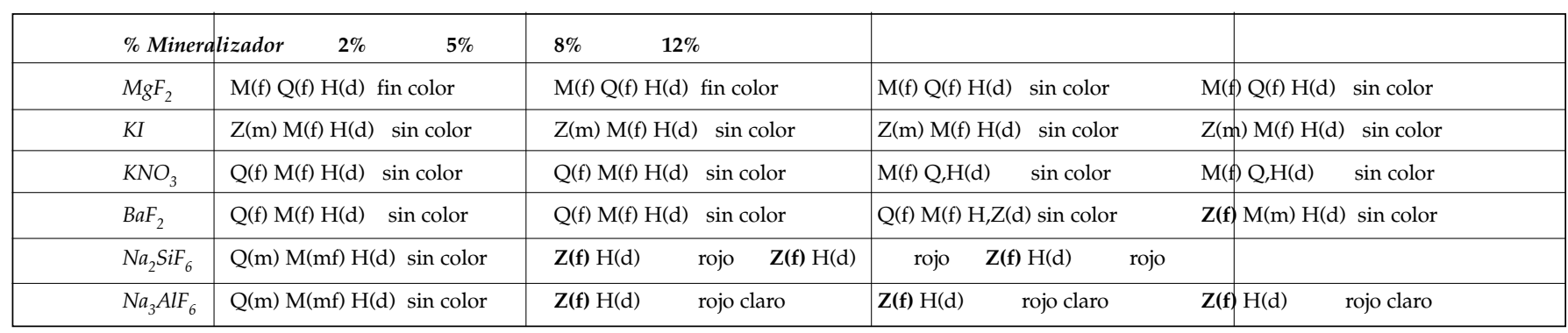

Fases cristalinas: $\mathrm{Q}$ (cuarzo, $\mathrm{SiO}_{2}$ ), $\mathrm{M}\left(\mathrm{ZrO}_{2}\right.$ monoclínica), $\mathrm{Z}$ (circón, $\mathrm{ZrSiO}_{4}$ ) y H (hematita, $\alpha-\mathrm{Fe}_{2} \mathrm{O}_{3}$ ). Intensidad de los picos de DRX: mf (muy fuerte), $\mathrm{f}$ (fuerte), $\mathrm{m}$ (media) y d (débil).

TABLA IV

NOMENClatura de LAS COMPOSICIONES Binarias $\left(\mathrm{SiO}_{2}\right)\left(\mathrm{ZrO}_{2}\right)_{0.97}\left(\mathrm{Fe}_{2} \mathrm{O}_{3}\right)_{0.15}(\mathrm{M} 1)_{0.70}(\mathrm{M} 2)_{0.30}$.

\begin{tabular}{|c|c|c|c|c|c|}
\hline$M 2 \rightarrow$ & $\mathbf{N a C l}$ & $\mathbf{N a}_{2} \mathrm{SiF}_{\mathbf{6}}$ & $\mathbf{B a F}_{2}$ & $\mathbf{N a}_{3} \mathrm{AlF}_{\mathbf{6}}$ & $\mathbf{K N O}_{3}$ \\
\hline$M 1=N a F$ & $1 \mathrm{~A}$ & $1 \mathrm{~B}$ & $1 \mathrm{C}$ & $1 \mathrm{D}$ & $1 \mathrm{E}$ \\
\hline$M 1=B a F_{2}$ & $2 \mathrm{~A}$ & $2 \mathrm{~B}$ & - & $2 \mathrm{D}$ & $2 \mathrm{E}$ \\
\hline
\end{tabular}


mineralizador empleado, ya sea simple (un sólo mineralizador ) o binario. En todas las composiciones se utilizaron los mismos precursores de $\mathrm{SiO}_{2}$ (cuarzo comercial de riqueza 95,5\% y $3,9 \mu \mathrm{m}$ de tamaño $\Phi_{50}$, suministrado por J.J. Navarro) y de $\mathrm{ZrO}_{2}$ (baddeleyita comercial de riqueza $95 \%$ y 5,6 $\mu \mathrm{m}$ de tamaño $\Phi_{50}$, suministrada por J.J. Navarro). Como precursor de hierro se utilizó o bien sulfato $\left(\mathrm{FeSO}_{4} \cdot 7 \mathrm{H}_{2} \mathrm{O}\right.$ de Panreac, fase única por DRX) o goetita, $\mathrm{FeO}(\mathrm{OH})$ (suministrado por J.J. Navarro, fase única por DRX). Los mineralizadores estudiados fueron: $\mathrm{NaF}, \mathrm{NaCl}, \mathrm{Na}_{2} \mathrm{SiF}_{6}, \mathrm{Na}_{3} \mathrm{AlF}_{6}, \mathrm{KI}, \mathrm{MgF}_{2}, \mathrm{BaF}_{2}$ y $\mathrm{KNO}_{3}$ (todos ellos de J.J. Navarro).

Las cantidades correspondientes de cada precursor y componente del sistema mineralizador fueron molturadas y homogeneizadas conjuntamente en unos molinos de bolas del tipo planetario y con bolas de alúmina, en medio dispersante de acetona. La acetona residual se eliminó por evaporación y los polvos secos resultantes fueron finalmente homogeneizados en un mortero de ágata. las mezclas precursoras así preparadas fueron sometidas a tratamiento térmico en un horno eléctrico hasta una temperatura máxima de calcinación comprendida entre 850 y $1100^{\circ} \mathrm{C}$, con tiempos de calentamiento y de retención a esta temperatura de 2 horas.

La calcinación de las muestras se realizó en crisoles silicoaluminosos, llenándolos en su totalidad y prensando manualmente el material en su interior, e invirtiéndolos sobre una tapa del mismo material.

Los polvos calcinados a las distintas temperaturas fueron caracterizados por difracción de rayos $\mathrm{X}$ (DRX), por el método del polvo, en un difractómetro Siemens utilizando radiación $\mathrm{K} \alpha$ de $\mathrm{Cu}$ y filtro de $\mathrm{Ni}$, analizándose la evolución de las fases cristalinas presentes. Con el fin de evaluar el rendimiento colorimétrico obtenido, los pigmentos sintetizados fueron lavados (se realizó un lavado estándar ácido-reductor de $1 \mathrm{~g}$ de cada

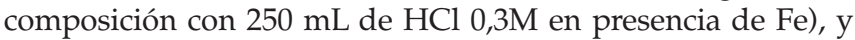
posteriormente esmaltados (con 5\% de color) sobre soportes cerámicos convencionales, utilizando un vidriado cristalino (transparente) comercial de monococción porosa. Los parámetros colorimétricos $L^{*} a^{*} b^{*}$ de las muestras esmaltadas que desarrollaron mejores coloraciones fueron medidos en un espectrofotómetro Perkin-Elmer, siguiendo el método CIE$\mathrm{L}^{*} \mathrm{a}^{*} \mathrm{~b}^{*}$ recomendado por la CIE (Comission Internationales de $l^{\prime}$ Eclairage) (17). En este sistema $L^{*}$ es una medida de la claridad - negro (0), blanco (100) - $a^{*}$ se corresponde con el eje que va del verde (-) al rojo (+), y $b^{*}$ es el eje del azul (-) al amarillo $(+)$.

Por otra parte, muestras representativas (en polvo o dispersiones en el esmalte) fueron también caracterizadas por microscopía óptica (en un microscopio óptico Olympus $\mathrm{BH}$ 2UMA) para confirmar la hipótesis de encapsulación de la hematita como origen del color. Finalmente, una de las composiciones pigmentantes seleccionadas como óptimas fue caracterizada micro-estructuralmente por microscopía electrónica de barrido (MEB) en un microscopio electrónico Leo-440i provisto de microanálisis de rayos $\mathrm{X}$ por dispersión de energías (AXDE).

TABLA V

EVOLUCIÓN CON LA TEMPERATURA DE CALCINACIÓN DE LAS FASES CRISTALINAS Y PARÁMETROS COLORIMÉTRICOS EN LAS COMPOSICIONES DE LA SERIE BINARIA 1A-1E $\left(\mathrm{SiO}_{2}\right)\left(\mathrm{ZrO}_{2}\right)_{0.97}\left(\mathrm{Fe}_{2} \mathrm{O}_{3}\right)_{0.15}(\mathrm{NaF})_{0.70}(\mathrm{M} 2)_{0.30}$, UTILIZANDO $\mathrm{FeSO}_{4} \cdot 7 \mathrm{H}_{2} \mathrm{O}$ COMO FUENTE DE HIERRO.

\begin{tabular}{|c|c|c|c|c|c|c|}
\hline muestra $1 \mathrm{~A}$ & $850^{\circ} \mathrm{C}$ & $900^{\circ} \mathrm{C}$ & $950^{\circ} \mathrm{C}$ & $1000^{\circ} \mathrm{C}$ & $1050^{\circ} \mathrm{C}$ & $1100^{\circ} \mathrm{C}$ \\
\hline$D R X$ & $\mathrm{Z}(\mathrm{mf}), \mathrm{M}, \mathrm{H}(\mathrm{md})$ & $\mathrm{Z}(\mathrm{mf}), \mathrm{M}, \mathrm{H}(\mathrm{md})$ & $\mathrm{Z}(\mathrm{mf}), \mathrm{M}, \mathrm{H}(\mathrm{md})$ & $\mathrm{Z}(\mathrm{mf}), \mathrm{M}, \mathrm{H}(\mathrm{md})$ & $\mathrm{Z}(\mathrm{mf}), \mathrm{M}, \mathrm{H}(\mathrm{md})$ & $\mathrm{Z}(\mathrm{mf}), \mathrm{M}, \mathrm{H}(\mathrm{md})$ \\
\hline$L^{*}$ & 61,4 & 57,0 & 61,6 & 56,0 & 52,9 & 53,0 \\
\hline$a^{*}$ & 25,4 & 28,3 & 24,9 & 28,0 & 25,5 & 26,5 \\
\hline$b^{*}$ & 26,5 & 24,3 & 27,0 & 24,1 & 16,4 & 20,2 \\
\hline muestra $1 B$ & $850^{\circ} \mathrm{C}$ & $900^{\circ} \mathrm{C}$ & $950^{\circ} \mathrm{C}$ & $1000^{\circ} \mathrm{C}$ & $1050^{\circ} \mathrm{C}$ & $1100^{\circ} \mathrm{C}$ \\
\hline$D R X$ & $\mathrm{Z}(\mathrm{mf}), \mathrm{M}, \mathrm{H}(\mathrm{md})$ & $\mathrm{Z}(\mathrm{mf}), \mathrm{M}, \mathrm{H}(\mathrm{md})$ & $\mathrm{Z}(\mathrm{mf}), \mathrm{M}, \mathrm{H}(\mathrm{md})$ & $\mathrm{Z}(\mathrm{mf}), \mathrm{M}, \mathrm{H}(\mathrm{md})$ & $\mathrm{Z}(\mathrm{mf}), \mathrm{M}, \mathrm{H}(\mathrm{md})$ & $\mathrm{Z}(\mathrm{mf}), \mathrm{M}, \mathrm{H}(\mathrm{md})$ \\
\hline$L^{*}$ & 60,2 & 56,8 & 56,7 & 54,1 & 54,9 & 53,1 \\
\hline$a^{*}$ & 26,6, & 28,5 & 28,4 & 27,7 & 26,6 & 25,3 \\
\hline$b^{*}$ & 25,4 & 22,6 & 22,4 & 19,4 & 20,2 & 17,7 \\
\hline muestra $1 C$ & $850^{\circ} \mathrm{C}$ & $900^{\circ} \mathrm{C}$ & $950^{\circ} \mathrm{C}$ & $1000^{\circ} \mathrm{C}$ & $1050^{\circ} \mathrm{C}$ & $1100^{\circ} \mathrm{C}$ \\
\hline$D R X$ & $\mathrm{Z}(\mathrm{mf}) \mathrm{B}, \mathrm{M}, \mathrm{H}(\mathrm{md})$ & $\mathrm{Z}(\mathrm{mf}) \mathrm{B}, \mathrm{M}, \mathrm{H}(\mathrm{md})$ & $\mathrm{Z}(\mathrm{mf}) \mathrm{B}, \mathrm{M}, \mathrm{H}(\mathrm{md})$ & $\mathrm{Z}(\mathrm{mf}) \mathrm{B}, \mathrm{M}, \mathrm{H}(\mathrm{md})$ & $\mathrm{Z}(\mathrm{mf}) \mathrm{B}, \mathrm{M}, \mathrm{H}(\mathrm{md})$ & $\mathrm{Z}(\mathrm{mf}) \mathrm{B}, \mathrm{M}, \mathrm{H}(\mathrm{md})$ \\
\hline$L^{*}$ & 62,0 & 58,0 & 61,0 & - & 59,2 & 57,2 \\
\hline$a^{*}$ & 24,1 & 26,7 & 24,8 & - & 24,9 & 24,1 \\
\hline$b^{*}$ & 27,7 & 25,3 & 27,2 & - & 24,8 & 21,8 \\
\hline muestra 1D & $850^{\circ} \mathrm{C}$ & $900^{\circ} \mathrm{C}$ & $950^{\circ} \mathrm{C}$ & $1000^{\circ} \mathrm{C}$ & $1050^{\circ} \mathrm{C}$ & $1100^{\circ} \mathrm{C}$ \\
\hline$D R X$ & $\mathrm{Z}(\mathrm{mf}), \mathrm{M}, \mathrm{H}(\mathrm{md})$ & $\mathrm{Z}(\mathrm{mf}), \mathrm{M}, \mathrm{H}(\mathrm{md})$ & $\mathrm{Z}(\mathrm{mf}), \mathrm{M}, \mathrm{H}(\mathrm{md})$ & $\mathrm{Z}(\mathrm{mf}), \mathrm{M}, \mathrm{H}(\mathrm{md})$ & $\mathrm{Z}(\mathrm{mf}), \mathrm{M}, \mathrm{H}(\mathrm{md})$ & $\mathrm{Z}(\mathrm{mf}), \mathrm{M}, \mathrm{H}(\mathrm{md})$ \\
\hline$L^{*}$ & 60,9 & 58,6 & 59,6 & 55,0 & 54,8 & 53,9 \\
\hline$a^{*}$ & 26,3 & 28,3 & 27,3 & 28,5 & 27,4 & 26,8 \\
\hline$b^{*}$ & 27,0 & 25,7 & 26,1 & 22,9 & 23,4 & 21,9 \\
\hline muestra 1E & $850^{\circ} \mathrm{C}$ & $900^{\circ} \mathrm{C}$ & $950^{\circ} \mathrm{C}$ & $1000^{\circ} \mathrm{C}$ & $1050^{\circ} \mathrm{C}$ & $1100^{\circ} \mathrm{C}$ \\
\hline$D R X$ & $\mathrm{Z}(\mathrm{mf}), \mathrm{M}, \mathrm{Q}(\mathrm{md})$ & $\mathrm{Z}(\mathrm{mf}), \mathrm{M}, \mathrm{Q}(\mathrm{md})$ & $\mathrm{Z}(\mathrm{mf}), \mathrm{M}, \mathrm{Q}(\mathrm{md})$ & $\mathrm{Z}(\mathrm{mf}), \mathrm{M}, \mathrm{Q}(\mathrm{md})$ & $\mathrm{Z}(\mathrm{mf}), \mathrm{M}, \mathrm{Q}(\mathrm{md})$ & $\mathrm{Z}(\mathrm{mf}), \mathrm{M}, \mathrm{Q}(\mathrm{md})$ \\
\hline$L^{*}$ & 76,4 & 60,4 & 59,9 & 56,6 & 57,8 & 55,3 \\
\hline$a^{*}$ & 10,4 & 27,3 & 27,9 & 29,8 & 28,6 & 28,1 \\
\hline$b^{*}$ & 21,3 & 27,1 & 28,1 & 26,1 & 27,2 & 24,2 \\
\hline
\end{tabular}

Fases cristalinas: $\mathrm{Q}$ (cuarzo, $\mathrm{SiO}_{2}$ ), $\mathrm{M}\left(\mathrm{ZrO}_{2}\right.$ monoclínica), $\mathrm{Z}$ (circón, $\mathrm{ZrSiO}_{4}$ ), $\mathrm{H}$ (hematita, $\alpha-\mathrm{Fe}_{2} \mathrm{O}_{3}$ ) y B (barita, BaSO $\mathrm{O}_{4}$ ). Intensidad de los picos de DRX: mf (muy fuerte) y md (muy débil).

Las medidas colorimétricas $\left(\mathrm{L}^{*} \mathrm{a}^{*} \mathrm{~b}^{*}\right)$ corresponden a las muestras esmaltadas (5\% color) con un vidriado cristalino convencional. 


\section{RESULTADOS Y DISCUSIÓN}

\subsection{Optimización de la adición de un sistema mineralizador} simple

La primera fase del estudio consistió en analizar la adición de un sistema mineralizador simple (compuesto por un único agente mineralizador) utilizando dos precursores diferentes de Fe (sulfato y goetita) y seleccionando aquellos mineralizadores con un efecto óptimo sobre el desarrollo del pigmento rosa coral.

\subsubsection{CON SULFATO DE HIERRO $\left(\mathrm{FeSO}_{4} \cdot 7 \mathrm{H}_{2} \mathrm{O}\right)$}

Empleando sulfato de hierro (heptahidrato) como precursor se prepararon composiciones de estequiometría $\left(\mathrm{SiO}_{2}\right)$ $\left(\mathrm{ZrO}_{2}\right)_{0,97}\left(\mathrm{Fe}_{2} \mathrm{O}_{3}\right)_{0,15}$ (Mineralizador) ${ }_{\mathrm{x}^{\prime}}$ añadiéndose en cada caso un 3\% en peso de uno solo de los siguientes mineralizadores: $\mathrm{NaF}, \mathrm{NaCl}, \mathrm{KNO}_{3}, \mathrm{BaF}_{2}, \mathrm{Na}_{3} \mathrm{AlF}_{6}$ y $\mathrm{Na}_{2} \mathrm{SiF}_{6}$. También se preparó una composición no mineralizada como referencia.

Atendiendo a la caracterización realizada por DRX (ver Tabla I), la reactividad de las muestras resultó ser muy diferente en función del mineralizador utilizado. En la composición no mineralizada, la calcinación entre 850 y $1100^{\circ} \mathrm{C}$ no permitió el desarrollo de circón, detectándose únicamente la presencia de las fases cristalinas originales (circona monoclínica, cuarzo y hematita). Por el contrario, las muestras mineralizadas con $\mathrm{NaF}, \mathrm{NaCl}$ y $\mathrm{BaF}_{2}$ destacaron por su mayor reactividad: el empleo de $\mathrm{NaF}$ dio lugar a un desarrollo completo de circón ya a $850^{\circ} \mathrm{C}$, con $\mathrm{NaCl}$ también se detectó circón a esta

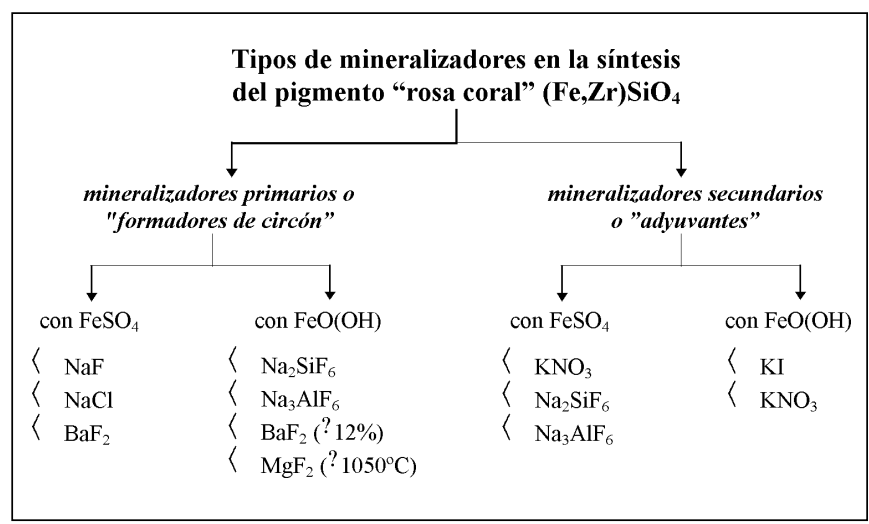

Figura 2. Clasificación de los mineralizadores ensayados en función de su efecto sobre el desarrollo de circón y la coloración, en la síntesis del pigmento de rosa coral de hierro-circón.

temperatura $\left(850^{\circ} \mathrm{C}\right.$ ) pero con un desarrollo parcial (picos de intensidad media), mientras que con $\mathrm{BaF}_{2}$ también se obtuvo un desarrollo completo de circón, aunque a partir de $900^{\circ} \mathrm{C}$. En esta composición se detectó también la cristalización de una pequeña cantidad de $\mathrm{BaSO}_{4}$ (barita, ASTM 24-1035) en todo el intervalo de temperaturas.

En cambio, las muestras mineralizadas con $\mathrm{Na}_{3} \mathrm{AlF}_{6}$ $\mathrm{Na}_{2} \mathrm{SiF}_{6}$ y $\mathrm{KNO}_{3}$ mostraron una reactividad inferior, no formándose circón en ninguna de las composiciones. En el caso del $\mathrm{KNO}_{3}$ se apreció una disminución progresiva en la intensidad de los picos de difracción de cuarzo, sin observarse de forma paralela una cristalización de circón, lo cual podría deberse a la formación de una fase vítrea con $\mathrm{SiO}_{2}$ y $\mathrm{K}_{2} \mathrm{O}$, que

TABLA VI

FASES CRISTALINAS DETECTADAS POR DRX A LAS DISTINTAS TEMPERATURAS DE CALCINACIÓN EN LAS COMPOSICIONES DE LA SERIE BINARIA 2, (SiO 2 ) $\left(\mathrm{ZrO}_{2}\right)_{0.97}\left(\mathrm{Fe}_{2} \mathrm{O}_{3}\right)_{0.15}\left(\mathrm{BaF}_{2}\right)_{0.70}(\mathrm{M} 2)_{0.30}$, PREPARADAS CON FeSO ${ }_{4} \cdot 7 \mathrm{H}_{2} \mathrm{O}$ COMO FUENTE DE HIERRO.

\begin{tabular}{|c|c|c|c|c|c|c|}
\hline Muestra / $T^{o} \mathrm{C}$ & $850^{\circ} \mathrm{C}$ & $900^{\circ} \mathrm{C}$ & $950^{\circ} \mathrm{C}$ & $1000^{\circ} \mathrm{C}$ & $1050^{\circ} \mathrm{C}$ & $1100^{\circ} \mathrm{C}$ \\
\hline $2 A$ & $\mathrm{Z}(\mathrm{mf}) \mathrm{B}, \mathrm{M}, \mathrm{H}(\mathrm{d})$ & $\mathrm{Z}(\mathrm{mf}) \mathrm{B}, \mathrm{M}, \mathrm{H}(\mathrm{d})$ & $\mathrm{Z}(\mathrm{mf}) \mathrm{B}, \mathrm{M}, \mathrm{H}(\mathrm{d})$ & $\mathrm{Z}(\mathrm{mf}) \mathrm{B}, \mathrm{M}, \mathrm{H}(\mathrm{d})$ & $\mathrm{Z}(\mathrm{mf}) \mathrm{B}, \mathrm{M}, \mathrm{H}(\mathrm{d})$ & $\mathrm{Z}(\mathrm{mf}) \mathrm{B}, \mathrm{M}, \mathrm{H}(\mathrm{d})$ \\
\hline $2 B$ & $\mathrm{Z}(\mathrm{mf}) \quad \mathrm{B}, \mathrm{H}(\mathrm{d})$ & $\mathrm{Z}(\mathrm{mf}) \quad \mathrm{B}, \mathrm{H}(\mathrm{d})$ & $\mathrm{Z}(\mathrm{mf}) \quad \mathrm{B}, \mathrm{H}(\mathrm{d})$ & $\mathrm{Z}(\mathrm{mf}) \quad \mathrm{B}, \mathrm{H}(\mathrm{d})$ & $\mathrm{Z}(\mathrm{mf}) \quad \mathrm{B}, \mathrm{H}(\mathrm{d})$ & $\mathrm{Z}(\mathrm{mf}) \quad \mathrm{B}, \mathrm{H}(\mathrm{d})$ \\
\hline $2 D$ & $\mathrm{Z}(\mathrm{mf}) \mathrm{B}, \mathrm{M}, \mathrm{H}(\mathrm{d})$ & $\mathrm{Z}(\mathrm{mf}) \mathrm{B}, \mathrm{M}, \mathrm{H}(\mathrm{d})$ & $\mathrm{Z}(\mathrm{mf}) \mathrm{B}, \mathrm{M}, \mathrm{H}(\mathrm{d})$ & $\mathrm{Z}(\mathrm{mf}) \mathrm{B}, \mathrm{M}, \mathrm{H}(\mathrm{d})$ & $\mathrm{Z}(\mathrm{mf}) \mathrm{B}, \mathrm{M}, \mathrm{H}(\mathrm{d})$ & $\mathrm{Z}(\mathrm{mf}) \mathrm{B}, \mathrm{M}, \mathrm{H}(\mathrm{d})$ \\
\hline $2 E$ & $\mathrm{Z}(\mathrm{m}) \mathrm{B}, \mathrm{M}, \mathrm{Q}, \mathrm{H}(\mathrm{md})$ & $\mathrm{Z}(\mathrm{m}) \mathrm{B}, \mathrm{M}, \mathrm{Q}, \mathrm{H}(\mathrm{md})$ & $\mathrm{Z}(\mathrm{m}) \mathrm{B}, \mathrm{M}, \mathrm{Q}, \mathrm{H}(\mathrm{md})$ & $\mathrm{Z}(\mathrm{m}) \mathrm{B}, \mathrm{M}, \mathrm{Q}, \mathrm{H}(\mathrm{md})$ & $\mathrm{Z}(\mathrm{m}) \mathrm{B}, \mathrm{M}, \mathrm{Q}, \mathrm{H}(\mathrm{md})$ & $\mathrm{Z}(\mathrm{m}) \mathrm{B}, \mathrm{M}, \mathrm{Q}, \mathrm{H}(\mathrm{md})$ \\
\hline
\end{tabular}

Fases cristalinas: $\mathrm{Q}$ (cuarzo, $\mathrm{SiO}_{2}$ ), $\mathrm{M}\left(\mathrm{ZrO}_{2}\right.$ monoclínica), $\mathrm{Z}$ (circón, $\mathrm{ZrSiO}_{4}$ ), $\mathrm{H}$ (hematita, $\alpha-\mathrm{Fe}_{2} \mathrm{O}_{3}$ ) y B (barita, BaSO $\mathrm{B}_{4}$. Intensidad de los picos de DRX: mf (muy fuerte), $\mathrm{m}$ (media), d (débil) y md (muy débil).

TABLA VII

FASES CRISTALINAS Y PARÁMETROS COLORIMÉTRICOS CORRESPONDIENTES A LAS COMPOSICIONES ÓPTIMAS.

\begin{tabular}{|c|c|c|c|}
\hline Composición & Temperatura ( $\left.{ }^{\circ} \mathrm{C}\right)$ & DRX & $\mathbf{L}^{*} / \mathbf{a}^{*} / \mathbf{b}^{*}$ \\
\hline $1 \mathrm{~A}(\mathrm{M} 2=\mathrm{NaCl})$ & 900 & $\mathrm{Z}(\mathrm{mf}) \mathrm{M}, \mathrm{H}(\mathrm{md})$ & $57,0 / 28,3 / 24,3$ \\
\hline $1 B\left(\mathrm{M} 2=\mathrm{Na}_{2} \mathrm{SiF}_{6}\right)$ & 900 & $\mathrm{Z}(\mathrm{mf}) \mathrm{M}, \mathrm{H}(\mathrm{md})$ & $56,8 / 28,5 / 22,6$ \\
\hline $1 B\left(\mathrm{M} 2=\mathrm{Na}_{2} \mathrm{SiF}_{6}\right)$ & 950 & $\mathrm{Z}(\mathrm{mf}) \mathrm{M}, \mathrm{H}(\mathrm{md})$ & $56,7 / 28,4 / 22,4$ \\
\hline $1 D\left(\mathrm{M} 2=\mathrm{Na}_{3} \mathrm{AlF}_{6}\right)$ & 1000 & $\mathrm{Z}(\mathrm{mf}) \mathrm{M}, \mathrm{H}(\mathrm{md})$ & $55,0 / 28,5 / 22,9$ \\
\hline $1 E\left(\mathrm{M} 2=\mathrm{KNO}_{3}\right)$ & 1000 & $\mathrm{Z}(\mathrm{mf}) \mathrm{M}, \mathrm{Q}(\mathrm{md})$ & $56,6 / 29,8 /, 26,1$ \\
\hline $1 E\left(\mathrm{M} 2=\mathrm{KNO}_{3}\right)$ & 1050 & $\mathrm{Z}(\mathrm{mf}) \mathrm{M}, \mathrm{Q}(\mathrm{md})$ & $57,8 / 28,6 / 27,2$ \\
\hline
\end{tabular}

Fases cristalinas: $\mathrm{M}\left(\mathrm{ZrO}_{2}\right.$ monoclínica), $\mathrm{Z}$ (circón, $\left.\mathrm{ZrSiO}_{4}\right)$ y H (hematita, $\alpha-\mathrm{Fe}_{2} \mathrm{O}_{3}$ ). Intensidad de los picos de DRX: mf (muy fuerte) y md (muy débil). 
de algún modo inhibiría el desarrollo del circón. Asimismo, sólo en el caso de la composición mineralizada con $\mathrm{KNO}_{3}$ se constató la presencia de una pequeña cantidad de circona tetragonal.

Por lo que respecta al rendimiento colorimétrico, y como era de esperar, sólo las composiciones con desarrollo de circón dieron lugar a coloraciones rojizas: con $\mathrm{NaCl}$ sólo se obtuvo una coloración rojiza clara a $1100^{\circ} \mathrm{C}$, con $\mathrm{BaF}_{2}$ los pigmentos eran más rojos al aumentar la temperatura de calcinación, mientras que con $\mathrm{NaF}$ las composiciones calcinadas entre 900 y $1000^{\circ} \mathrm{C}$ fueron las que desarrollaron un color rojo más intenso.

\subsubsection{CON GOETITA (FeO(OH))}

Empleando goetita (heptahidrato) como precursor se prepararon en primer lugar composiciones de estequiometría $\left(\mathrm{SiO}_{2}\right)$ $\left(\mathrm{ZrO}_{2}\right)_{0,97}\left(\mathrm{Fe}_{2} \mathrm{O}_{3}\right)_{0,15}$ (Mineralizador $)_{x^{\prime}}$ añadiéndose en cada caso un $2 \%$ en peso de uno solo de los siguientes mineralizadores: $\mathrm{MgF}_{2}, \mathrm{KI}, \mathrm{KNO}_{3}, \mathrm{BaF}_{2}, \mathrm{Na}_{3} \mathrm{AlF}_{6} \mathrm{y} \mathrm{Na}_{2} \mathrm{SiF}_{6}$. También se preparó una composición no mineralizada como referencia.

De forma similar que empleando sulfatos, las muestras con goetita fueron calcinadas entre 850 y $1100^{\circ} \mathrm{C}$, analizándose la influencia de los distintos mineralizadores sobre el desarrollo del pigmento a las distintas temperaturas (ver resultados de DRX en la Tabla II). Dada la baja reactividad observada, se realizó un segundo estudio utilizando esta vez cantidades mayores de los distintos mineralizadores $(2,5,8$ y $12 \%$ en peso) y calcinando las composiciones a una temperatura intermedia $\left(950^{\circ} \mathrm{C}\right)$, cuyos resultados se muestran en la Tabla III.

En el caso de las composiciones con una baja adición de mineralizador (2\%), sólo el empleo de $\mathrm{MgF}_{2}$ permitió el desarrollo de circón (ver Tabla II), aunque a temperaturas elevadas (a partir de $1050^{\circ} \mathrm{C}$ ). Sin embargo, con este mineralizador no se pudo formar circón calcinando a $950^{\circ} \mathrm{C}$ con adiciones superiores del mismo. En este sentido, el análisis de los resultados obtenidos a $950^{\circ} \mathrm{C}$ con un mayor contenido de mineralizador (Tabla III), permiten agrupar a los mineralizadores en tres categorías diferentes, considerando su efecto sobre la formación de circón y sobre la eficiencia del encapsulamiento o rendimiento colorimétrico:

i) agentes mineralizadores fuertes o promotores del color $\left(\mathrm{Na}_{3} \mathrm{AlF}_{6}\right.$ y $\mathrm{Na}_{2} \mathrm{SiF}_{6}$ ): estos compuestos inducen la cristalización del circón con adiciones iguales o superiores al $5 \%$ en peso. Con $\mathrm{Na}_{3} \mathrm{AlF}_{6}$, conforme aumenta la cantidad de mineralizador la coloración rojiza disminuye su intensidad ligeramente, mien- tras que el fluorosilicato promueve mejor el desarrollo del color.

ii) agentes mineralizadores y promotores del color débiles $\left(\mathrm{BaF}_{2}\right.$ y $\left.M g F_{2}\right)$ : con $\mathrm{BaF}_{2}$ sólo se obtiene circón con una alta adición $(12 \%)$, aunque no se consigue encapsular eficazmente a la hematita ya que no se desarrolla coloración. En cambio, tal y como se ha comentado, el $\mathrm{MgF}_{2}$ permite desarrollar el pigmento rosa coral, aunque sólo a elevadas temperaturas (desde $\left.1050^{\circ} \mathrm{C}\right)$.

iii) agentes mineralizadores adyuvantes $\left(\mathrm{KNO}_{3}\right.$ y $\left.\mathrm{KI}\right)$ : con ambos compuestos no se obtuvo circón en ninguna de las composiciones, pero, a pesar de ello, estos agentes podrían tener un efecto positivo sobre la síntesis, ya que por una parte descomponen a temperaturas relativamente bajas generando fuertes emisiones, y por otro lado pueden actuar como agentes oxidantes o reductores sobre el sistema Fe(II)-Fe(III); en todo caso, su acción mineralizadora estará supeditada a que el circón cristalice en torno a las temperaturas a las cuales tienen lugar estos procesos.

\subsection{Optimización de la adición de un sistema mineralizador binario}

En una segunda fase del estudio, a partir de los resultados obtenidos con la adición simple de los distintos mineralizadores, se formularon composiciones mineralizadas con un sistema binario (formado por dos agentes mineralizadores). A fin de facilitar la formulación de estas composiciones, los distintos mineralizadores ensayados anteriormente fueron agrupados en dos familias diferentes, tanto en el caso del empleo de sulfatos como con goetita como precursor de hierro (ver Figura 2):

i) mineralizadores primarios o "formadores de circón": aquellos compuestos con un efecto favorable sobre la formación del circón y, por tanto, sobre el desarrollo del color.

ii) mineralizadores secundarios o adyuvantes: todos aquellos compuestos que, no siendo capaces de formar circón en su adición simple, ejercen un papel colateral controlando aspectos secundarios pero también importantes en la síntesis del pigmento, como son los procesos de cristalización (regulando su tamaño) y de oclusión de la hematita en los cristales neoformados de circón.

Teniendo en cuenta esta clasificación, se prepararon dos series diferentes ( 1 y 2 ) con un sistema mineralizador binario (ver nomenclatura en la Tabla IV), con la estequiometría global

TABLA VIII

ANÁLISIS POR MICROSCOPÍA ÓPTICA DE LAS DISPERSIONES DE COLORES EN EL VIDRIADO CERÁMICO CRISTALINO (TRANSPARENTE).

\begin{tabular}{|c|c|c|c|}
\hline Composición & granulos \% & color & tamaño medio $(\mu \mathrm{m})$ \\
\hline $1 A-1000^{\circ} \mathrm{C}$ & 25 & naranja & 20 \\
\hline $1 A-1050^{\circ} \mathrm{C}$ & 5 & rojo & 20 \\
\hline $1 B-1000^{\circ} \mathrm{C}$ & 20 & naranja & 25 \\
\hline $1 B-1050^{\circ} \mathrm{C}$ & 20 & naranja & 20 \\
\hline $1 D-1000^{\circ} \mathrm{C}$ & 35 & rojo & 20 \\
\hline $1 D-1050^{\circ} \mathrm{C}$ & 40 & rojo & 20 \\
\hline $1 E-1000^{\circ} \mathrm{C}$ & 40 & naranja & 20 \\
\hline $1 E-1050^{\circ} \mathrm{C}$ & 25 & naranja claro & \\
\hline
\end{tabular}


$\left(\mathrm{SiO}_{2}\right)\left(\mathrm{ZrO}_{2}\right)_{0,97}\left(\mathrm{Fe}_{2} \mathrm{O}_{3}\right)_{0,15}(\mathrm{M} 1)_{0,70}(\mathrm{M} 2)_{0,30^{\prime}}$ donde como primer mineralizador (M1) se introdujo uno de los agentes "formadores de circón" ( $\mathrm{NaF}$ en la serie $1 \mathrm{~A}-1 \mathrm{E}$ y $\mathrm{BaF}_{2}$ en la serie 2A-2E), añadiéndose un segundo mineralizador (M2), ya sea primario o secundario, en menor concentración. Considerando los mejores resultados obtenidos, en cuanto a desarrollo del color, con la utilización de $\mathrm{FeSO}_{4} \cdot 7 \mathrm{H}_{2} \mathrm{O}$ como precursor de hierro, y el hecho de que este precursor tenga un uso más extendido en la producción industrial del pigmento estas dos series binarias fueron preparadas únicamente con sulfato de hierro. Como en los casos anteriores, las composiciones preparadas fueron calcinadas entre $850^{\circ} \mathrm{C}$ y $1100^{\circ} \mathrm{C}$ siguiendo el mismo tratamiento térmico.

En las tablas V y VI se resumen los resultados de la caracterización por DRX y colorimétrica (sólo la serie 1) correspondientes a las dos series binarias. Como hechos relevantes podemos destacar:

i) en todas las composiciones (excepto la $2 \mathrm{E}$, mineralizada con $\mathrm{BaF}_{2}-\mathrm{KNO}_{3}$ ) se obtuvo un buen desarrollo de circón, estando acompañada dicha fase de pequeñas cantidades de baddeleyita ( $\mathrm{ZrO}_{2}$ monoclínica), hematita, y en algunos casos cuarzo (muestras con $\mathrm{KNO}_{3}$ ) y sulfato de bario (muestras con $\mathrm{BaF}_{2}$ ).

ii) las composiciones de la serie 2 (con $\mathrm{BaF}_{2}$ como mineralizador primario) exhibieron unas coloraciones rojizas más claras (o menos intensas) una vez esmaltadas, desestimándose la medida de sus parámetros colorimétricos. En este sentido, conviene resaltar la mayor idoneidad del empleo de $\mathrm{NaF}$ como agente mineralizador primario, siendo más efectivo que el $\mathrm{BaF}_{2}$ en el desarrollo del pigmento.

iii) el mejor rendimiento colorimétrico se obtiene trabajando con los sistemas binarios $1 \mathrm{~B}\left(\mathrm{NaF}-\mathrm{Na}_{2} \mathrm{SiF}_{6}\right), 1 \mathrm{D}\left(\mathrm{NaF}_{-} \mathrm{Na}_{3} \mathrm{AlF}_{6}\right)$ y $1 \mathrm{E}\left(\mathrm{NaF}-\mathrm{KNO}_{3}\right)$. Las composiciones con $\mathrm{KNO}_{3}$ dan lugar a coloraciones más claras ( $L^{*}$ mayor) y más amarillas ( $b^{*}$ mayor). En la Tabla VII se resumen los resultados de difracción y colorimétricos obtenidos con las composiciones (y temperaturas de calcinación) seleccionadas como óptimas.

\subsection{Caracterización por microscopía óptica y electrónica}

Como último apartado del estudio, muestras representativas de la serie binaria $1 \mathrm{~A}-1 \mathrm{E}$ fueron caracterizadas por microscopía óptica con el objeto de confirmar la naturaleza encapsulada del pigmento. En la tabla VIII se muestran los resultados del análisis por microscopía óptica realizadas con las dispersiones de los pigmentos en el esmalte, en la que se indica la estimación realizada del porcentaje de gránulos coloreados (obtenido por análisis de imagen) presente en las composiciones, así como su color y tamaño medio. Los resultados confirmaron la existencia de una relación directa entre el porcentaje de gránulos coloreados (en principio, aquellos con hematita encapsulada) y la intensidad de la coloración rojiza de los esmaltados. Por ejemplo, este porcentaje disminuyó del 25 al 5\% en la composición 1A al aumentar la temperatura de calcinación de 1000 a $1050^{\circ} \mathrm{C}$, observándose de forma paralela una disminución de la coloración rojiza en las muestras esmaltadas (el parámetro a* disminuye de 28,0 a 25,5 ). La misma correlación entre ambos parámetros se observó en la composición 1E.

Según estos resultados, la pérdida de color rosa que se produce en algunos casos con el aumento de la temperatura, podría explicarse en base a un crecimiento excesivo de la hematita o del circón, que impedirían un encapsulamiento eficaz de la hematita, de acuerdo con el modelo propuesto en la

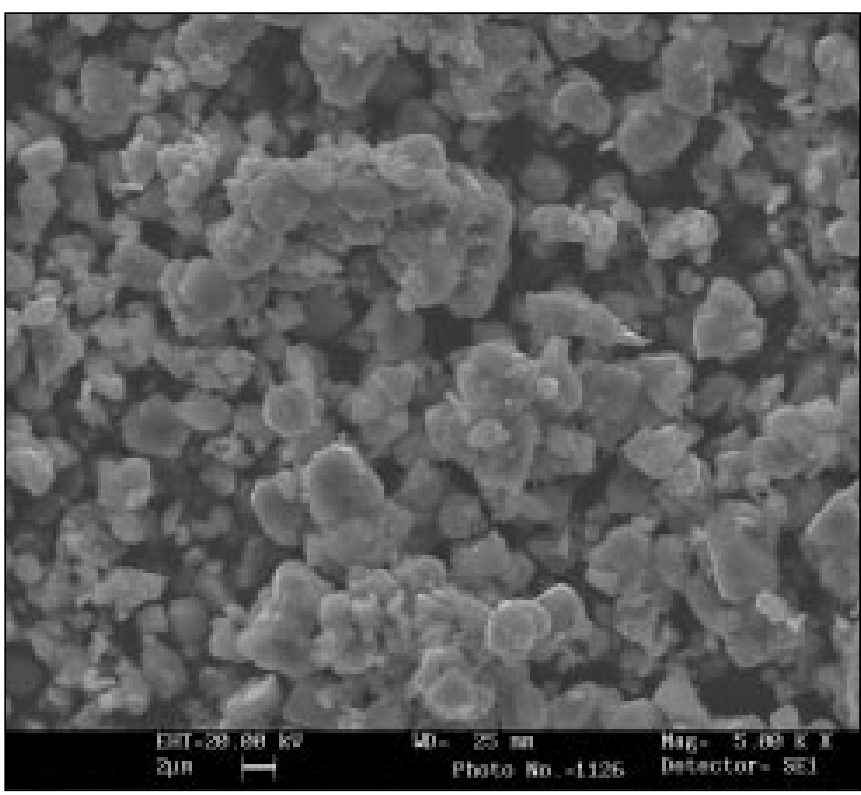

a)

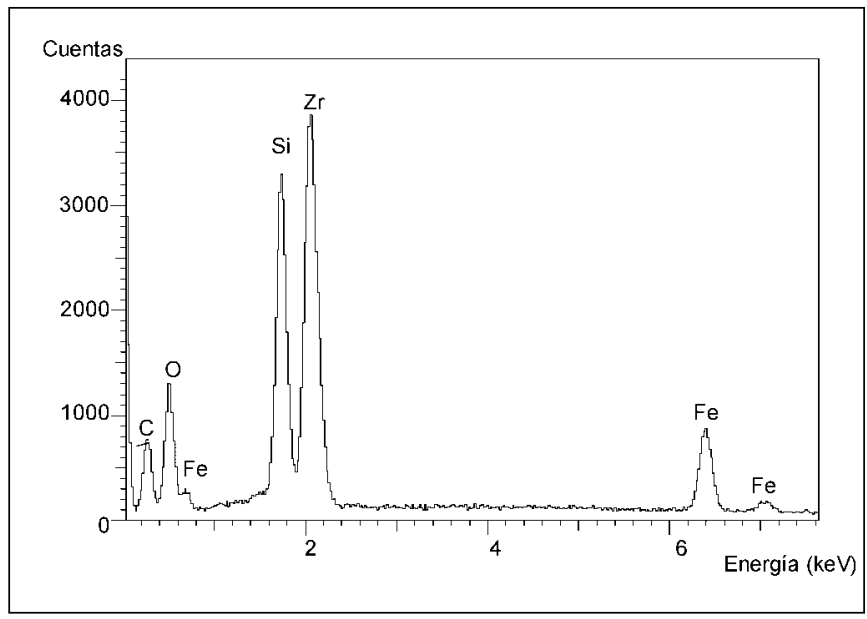

b)

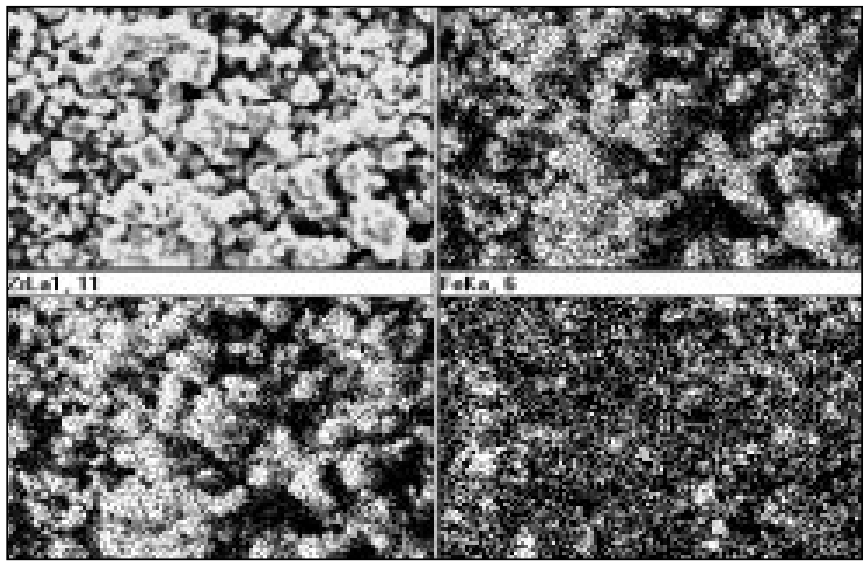

Figura 3. Micrografía de MEB (detector de secundarios, 5000 aumentos) de un detalle de la composición 1D calcinada a $1000^{\circ} \mathrm{C}$ (a), con los correspondientes espectro (b) y "mapping" (c) obtenidos por microanálisis de rayos $\mathrm{X}$ por dispersión de energías (AXDE). 
introducción (Figura 1). Estas partículas de hematita libre serían posteriormente eliminadas en el lavado del pigmento calcinado.

Asimismo, en observaciones por microscopía óptica realizadas con pigmentos no esmaltados (en polvo) representativos de las dos series (1D-1050 $0^{\circ} \mathrm{C}$ y $2 \mathrm{D}-1050^{\circ} \mathrm{C}$ ), pudo constatarse la mayor presencia de gránulos coloreados de rojo en la composición 1D, mientras que la composición 2D, que desarrolló una coloración más pobre una vez esmaltada, tenía una mayor porcentaje de gránulos no coloreados. La existencia de esta correlación entre el porcentaje de gránulos coloreados y el rendimiento colorimétrico obtenido, nos estaría confirmando de algún modo la hipótesis de encapsulación de la hematita como origen del color, tal y como se acepta ampliamente en la bibliografía.

Por último, una de las composiciones seleccionadas como óptima (1D- $\left.1000^{\circ} \mathrm{C}\right)$ fue también caracterizada microestructuralmente (ya lavada) por microscopía electrónica de barrido con microanálisis (MEB/AXDE). En la Figura 3a se presenta la imagen MEB obtenida (con el detector de electrones secundarios) de un detalle de dicha muestra, mientras que el espectro y "mapping" EDX correspondientes al mismo detalle pueden verse en las Figuras $3 b$ y $3 c$, respectivamente. El mapping EDX confirmó la distribución homogénea del Fe junto con el circón, de acuerdo con el modelo de oclusión propuesto, aunque también se apreció una pequeña cantidad de Fe segregado (como hematita libre), que habría resistido el lavado ácido-reductor. Según la imagen MEB, el circón se encuentra en forma de agregados redondeados de morfología irregular, con tamaños comprendidos entre 2 y $10 \mu \mathrm{m}$, junto con partículas dispersas de tamaño más pequeño $(1-2 \mu \mathrm{m})$.

\section{CONCLUSIONES}

(1) La adición de distintos agentes mineralizadores ha demostrado tener un comportamiento diferente en la síntesis del pigmento rosa coral de hierro-circón $(\mathrm{Fe}, \mathrm{Zr}) \mathrm{SiO}_{4}$, dependiendo del precursor de hierro utilizado (sulfato o goetita).

(2) Con sulfato de hierro, compuestos tales como $\mathrm{NaF}, \mathrm{NaCl}$ y $\mathrm{BaF}_{2}$ destacan como "mineralizadores formadores de circón": con una adición relativamente baja ( $3 \%$ en peso), las composiciones con $\mathrm{NaF}$ y $\mathrm{NaCl}$ ya desarrollan circón a $850^{\circ} \mathrm{C}$ (aunque siempre con circona monoclínica residual en el caso del $\mathrm{NaCl}$ ); por su parte, con $\mathrm{BaF}_{2}$ se obtiene un rendimiento de circón del $25 \%$ a $1100^{\circ} \mathrm{C}$.

(3) Con goetita $(\mathrm{FeOOH})$ el rendimiento colorimétrico obtenido es más pobre que con sulfato de hierro, destacando en este caso la mineralización con $\mathrm{Na}_{2} \mathrm{SiF}_{6} \mathrm{y} \mathrm{Na}_{3} \mathrm{AlF}_{6}$, que producen un buen desarrollo de circón a $950^{\circ} \mathrm{C}$ con adiciones del $5 \%$, mientras que el $\mathrm{BaF}_{2}$ y el $\mathrm{MgF}_{2}$ consiguen desarrollar circón con más dificultad (el primero a elevadas concentraciones, $12 \%$, y el segundo a elevadas temperaturas, $\left.1050^{\circ} \mathrm{C}\right)$.

(4) Otra serie de compuestos, que han sido denominados agentes "mineralizadores secundarios o adyuvantes" $\left(\mathrm{KNO}_{3}\right.$, $\mathrm{Na}_{2} \mathrm{SiF}_{6}$ y Na $\mathrm{NalF}_{6}$ con sulfato, y KI y $\mathrm{KNO}_{3}$ con goetita), no son capaces de desarrollar circón en su adición simple, pero podrían tener un efecto colateral en la síntesis del pigmen- to, regulando los procesos de crecimiento y oclusión de la hematita.

(5) Las composiciones mineralizadas con los sistemas binarios $1 \mathrm{~B}\left(\mathrm{NaF}-\mathrm{Na}_{2} \mathrm{SiF}_{6}\right), 1 \mathrm{D}\left(\mathrm{NaF}-\mathrm{Na}_{3} \mathrm{AlF}_{6}\right)$ y $1 \mathrm{E}\left(\mathrm{NaF}-\mathrm{KNO}_{3}\right)$ han sido seleccionadas como óptimas en la síntesis del pigmento, permitiendo la obtención de coloraciones rojizas más intensas.

(6) Las observaciones realizadas por microscopía óptica parecen confirmar la hipótesis de encapsulación de la hematita en los cristales de circón como origen del color, mostrando la existencia de una correlación entre el porcentaje de gránulos coloreados (con hematita ocluida) y la intensidad de los esmaltados.

\section{AGRADECIMIENTOS}

Mario Llusar muestra su agradecimiento a la Conselleria de Cultura, Educació i Ciència de la Generalitat Valenciana por el apoyo económico prestado con la concesión de la beca F.P.I.

\section{BIBLIOGRAFIA}

1. DRY COLORS MANUFACTURERS ASS., DCMA, "Classification and chemical description of the mixed metal inorganic coloured pigments", (2n ed., Washington DC, 1982).

2. T. Kendall, "Inorganic coloured pigments. Iron oxides looking for a rosy future", Industrial Minerals, 49-59 (febrero 1994).

3. G. Monrós, J. Carda, M. A. Tena, P. Escribano, M. Sales y J. Alarcón, "Different Kinds of Solids Solutions in the $\mathrm{V}_{2} \mathrm{O}_{5}-\mathrm{ZrSiO}_{4}-\mathrm{NaF}$ System by SolGel Processes and their Characterization", J. Europ. Ceram. Soc. 1177 (1993).

4. L. Marchesini, E. Ramous, "Sulla Structura dei Pigmenti Verdi e Bleu Turchese Contenenti Vanadio", Vetro Silic. 455 (1964).

5. T. Demiray, D.K. Nath, F.A. Hummel, "Zircon-vanadium blue pigment", J. Am. Ceram. Soc. 531 (1970).

6. V. I. Matkovith, P. M. Corbett, "Formation of zircon from zirconium dioxide and silicon dioxide in the presence of vanadium pentoxide", J. Am. Ceram. Soc. 44 [3] 128-130 (1961)

7. W.D. Kingery, H.K. Bowen and D.R. Uhlman, "Introduction to Ceramics", p. 314, 2nd edn. John Wiley and Sons, New York (1976).

8. M. A. Tena, G. Monrós, J. Carda, P. Escribano and J. Alarcón, "Efecto mineralizador del $\mathrm{V}_{2} \mathrm{O}_{5}$ en las transiciones de fase de pigmentos cerámicos", Bol. Soc. Esp. Ceram. Vidr. 29 177-179 (1990).

9. E. Cordoncillo, F. del Río, J. Carda, M. Llusar and P. Escribano, "Influence of Some Mineralizers in the Synthesis of Sphene-Pink Pigments", J. Europ. Ceram. Soc. 18 1115-1120 (1998)

10. R. A. Eppler, "Mechanism of Formation of Zircon Stains", J. Am. Ceram. Soc. 53 [8] 457-462 (1970).

11. R. A. Eppler, "Solid State Reacions in the Preparation of Zircon Stains", pp. 1021-1045 en Physics of Electronic Materials. Ed. L.L. Hench and D.B. Dove. Marcel Dekker, New York (1972)

12. K.M. Trappen and R. A. Eppler, "Reaction of Zirconia with Silica at the Stoichiometry of Zircon", J. Am. Ceram. Soc. 72 [6] 882-885 (1989).

13. A.C. Airey y W. Roberts, "Advances in Ceramic Colors", Ceram. Eng. Sci. Proc. 8 [11-12] 1168 (1987).

14. J. Lahuerta, "Colorantes Cerámicos Rojos basados en $\mathrm{Fe}_{2} \mathrm{O}_{3}$ ”, Tesis Doctoral, Universidad de Valencia, 1993.

15. Chi-Hang Li, D.R. Eppler y R.A. Eppler, "Iron Zircon Pigments", Ceram. Eng. Sci. Proc., 13 [1-2] 109 (1992).

16. P. Tartaj, T. González-Carreño, C.J. Serna y M. Ocaña, "Iron Zircon Pigments Prepared by Pyrolysis of Aerosols", J. of Solid State Chem. 128102 (1997).

17. CIE, "Reccommendations on Uniform Colour Spaces, Colour Difference Equations, Psychometrics Colour Terms" ",Suplement nำ of CIE Publ. №15 (E1-1.31) 1971, Bureau Central de la CIE, Paris, (1978).

Recibido: $29-12-98$

Aceptado: 21-1-99 